Commun. Math. Phys. 138, 207-208 (1991)

Communications in

Mathematical

Physics

(C) Springer-Verlag 1991

\title{
Erratum
}

\section{A "Transversal" Fundamental Theorem for Semi-Dispersing Billiards}

Commun. Math. Phys. 129, 535-560 (1990)

\author{
A. Krámli ${ }^{1}$, N. Simányi ${ }^{2}$, and D. Szász ${ }^{2}$ \\ 1 Computer and Automation Institute of the Hungarian Academy of Sciences, P.O. Box 127, \\ H-1364 Bundapest, Hungary \\ 2 Mathematical Institute of the Hungarian Academy of Sciences, P.O. Box 127, \\ H-1364 Budapest, Hungary
}

Received January 22, 1991

C. Liverani and M.P. Wojtkowski made a remark that the statement (ii) of our Lemma 2.13 is not necessarily true as formulated there. However, it remains valid if we use the norms of the operators $\left(D_{x, \Sigma}^{t}\right)^{-1},\left(D_{x, \Sigma}^{n}\right)^{-1}$ (cf. (2.8) and (2.9)) as acting in $\mathscr{T}_{x} \Sigma$ supplied with the configuration space norm $\|d q\|$ instead of the phase space Riemannian metric $\sqrt{(d q)^{2}+(d v)^{2}}$. This change has several consequences which are described in detail as follows:

1. In Definition 5.1, the remark between parentheses is not true, but it is not used later on.

2. In the formula (5.2) it can be noted that $1 \leqq \kappa_{n, \delta}(y) \leqq \kappa_{n, 0}(y)$.

3. In (ii) of Definition 5.1: the ball $B_{\delta}(-y)$ should be defined in terms of the degenerate configuration-space-metric $\|d q\|$.

4. Rethinking the proof of Lemma 5.4 we see that the construction of the local invariant manifolds does not use directly the function $z($.$) appearing in the$ definition of the sets $U_{n}^{b}$, but, instead, it works with another function $z_{\text {tub }}($.) which is just the original $z$-function given by Sinai and Chernov in S-Ch (1987). Recall that $z_{\text {tub }}(x)(x \in \partial M)$ is the supremum of the radii $r$ of all tubular neighborhoods $U_{r}$ of the projected trajectory segment $\pi\left(\left\{S^{t} x: 0 \leqq t \leqq \tau(x)\right\}\right)$ in the configuration space, for which the set $\left\{y \in M: p(y)=p(x)\right.$ and $\left.\pi(y) \in \bar{U}_{r}\right\}$ does not intersect the set of singular reflections. Notice that $z_{\text {tub }}($.$) is closely related to the metric \|d q\|$ being a Lyapunov one in the local orthogonal manifolds. A simple geometric argument shows that $z(x) \leqq z_{\text {tub }}(x)$.

The corrections of some other discovered errors are listed below:

a) The set treated in the second paragraph after Condition 2.1 has not only measure zero, but it is actually empty.

b) Convergence of the continued fraction (2.6) is proved in Lemma 1 of S-Ch (1982) for every $x \in M$ such that $t_{n} \rightarrow \infty$ as $n \rightarrow \infty$, a property valid for all phase points $x \in M^{*}$ because there are no trajectories with infinitely many collisions in a finite time interval. 
c) Just after Theorem 2.10: the function $l(x)$ is not upper semicontinuous, but lower semicontinuous.

d) In the proof of Lemma 2.13, when applying the flow $S^{-t_{+}}$to $S^{t+} x$, we may loose the validity of (ii). Instead of doing so, we can prove in small neighborhoods of $S^{t+} x$ the statement of the fundamental theorem which is invariant under the flow, thus the whole machinery can be transferred back to $x$.

e) In the part (b) of Definition 3.4: $w_{i}^{\delta} \in \partial M^{0}$ is to be written.

f) After Lemma 4.6 , in the definition of angle $\left(\mathscr{L}_{1}, \mathscr{L}_{2}\right)$ one has to write

$$
\sup _{v_{1} \in \mathscr{L}_{1}} \inf _{v_{2} \in \mathscr{L}_{2}} \text { angle }\left(v_{1}, v_{2}\right) \text {. }
$$

g) In the line before (5.10): the condition $G_{i}^{\delta} \in \mathscr{G}_{g}^{\delta}$ is to be canceled.

h) In the last but one line of Sect. 5: $\varepsilon_{2}$ should be written instead of $\varepsilon_{1}$.

The authors thank C. Liverani and M. Wojtkowski for their important remark.

\section{References}

[S-Ch] Sinai, Ya.G., Chernov, N.I.: Entropy of gas of hard spheres with respect to the group of time-space translations. Trudi Seminara Petrovskovo 8, 218-238 (1982)

[S-Ch] Sinai, Ya.G., Chernov, N.I.: Ergodic properties of some systems of 2-D discs and 3-D spheres. Usp. Mat. Nauk 42, 153-174 (1987)

Communicated by A. Jaffe 